\title{
A Strategy-Proof Multiunit Double Auction Mechanism
}

\author{
Pu Huang \\ CALD, School of Computer \\ Science \\ Carnegie Mellon University \\ Pittsburgh, PA 15213 \\ phuang@cs.cmu.edu
}

\author{
Alan Scheller-Wolf \\ Graduate School of Industrial \\ Administration \\ Carnegie Mellon University \\ Pittsburgh, PA 15213 \\ awolf@andrew.cmu.edu
}

\author{
Katia Sycara \\ School of Computer Science \\ Carnegie Mellon University \\ Pittsburgh, PA 15213 \\ katia@cs.cmu.edu
}

\begin{abstract}
We envision a future economy where e-markets will play an essential role as exchange hubs for commodities and services. Because of their flexibility, we anticipate multi-unit double auctions (MDAs) to play an important role in future's e-markets. In this paper, we present a multi-unit double auction mechanism which is strategy-proof with respect to reservation price, weakly budget-balanced and individually rational. In additions, by bounding the efficiency loss, we prove that our mechanism is alsoasymptotically efficient.
\end{abstract}

\section{Keywords}

electronic commerce, auction, mechanism design.

\section{INTRODUCTION}

Recent years have seen a growing interest among both academia and industry in Internet market-places. Enabled by modern network technologies, e-markets promise nearly friction-free information exchange, broad access to potential buyers and sellers, and almost perfect competition. Auctions are one of the most widely used exchange institutions in e-markets. Ebay, FreeMarkets, uBid, etc., to just name a few, all use auctions to organize their e-markets. Although many B2C e-markets, like Ebay, conduct one-side auctions, B2B e-markets, like FreeMarkets, predominately use multiunit double auctions because they can accommodate multiple agents trading multiple units, and thus are more proper in a B2B environment. In a double auction market, sellers and buyers submit asks and bids respectively. A transaction clears if a buyer's bid exceeds a seller's ask. Typically, a seller has multiple units for sale and a buyer also wants to purchase more than one unit. Therefore, a seller's ask may match several buyers' bids and a buyer's bid may satisfy several sellers' asks. The mechanism (or trading rule) of a double auction market must be able to process this sort of matching between multiple sellers and multiple buyers involving multiple units. Mechanism design is one of the most

Permission to make digital or hard copies of all or part of this work for personal or classroom use is granted without fee provided that copies are not made or distributed for profit or commercial advantage and that copies bear this notice and the full citation on the first page. To copy otherwise, to republish, to post on servers or to redistribute to lists, requires prior specific permission and/or a fee.

AAMAS'02, July 15-19, 2002, Bologna, Italy.

Copyright 2002 ACM 1-58113-480-0/02/0007 ...\$5.00. crucial aspects of double auction markets, because the mechanism not only directly defines the trading rules, but also implicitly defines the behavior of each participating agent. If we build software agents to trade in these markets, the mechanism also has great effect on the design of the architecture of these agents.

Our MDA mechanism is strategy-proof with respect to price, weakly budget-balanced, asymptotically efficient, and individually rational. Strategy-proofness means that revealing truthful private information is each agent's best strategy independent of what other agents are doing. often achieved by arranging some special form of payments between buyers and sellers. A mechanism is weak budget-balanced if all these payments sum to a positive number. An efficiency mechanism maximizes the total profit obtained by all participating agents. Our mechanism is asymptotically efficient, which means its efficiency increases to $100 \%$ as the number of agents increases. An agent will participate in an e-market only if it expects a nonnegative profit. Therefore, an MDA mechanism must also be designed to be individually rational, i.e., it attracts individual agents to voluntarily participate in the e-market because they expect nonnegative ex ante profits.

Voluminous game theory literature focuses on auction markets, specifically one-side auction markets where there is a monopoly with multiple buyers, or an oligopoly with multiple sellers. However, the literature on double auction market design is limited. Satterthwaite and Williams [4] were among the early researchers studying double auction markets. They designed a single-unit double auction (SDA) market where they simplified the analysis by eliminating the strategic behavior (mis-reporting one's true reservation price) on the sellers' side and showed that the difference between a buyer's bid and his reservation value went to zero in the limit as the number of traders grows. Thus the market converged to efficiency. In Satterthwaite and Williams, no third party was required to balance the market budget. Still in a SDA market, McAfee [3] allowed strategic behavior on both sides of the market and required a market maker to balance the budget. He proposed a strategy-proof mechanism and showed that the inefficiency converged to zero as the market became large in a weak sense, i.e., the surplus taken by the market maker was not counted as efficiency loss. Barbera and Jackson [1] characterized a set of strategy-proof mechanism for a multi-unit exchange market where every agent could choose to be a buyer or a seller. Their mechanism was not asymptotically efficient and required a third party to pre-specify a set of price proposals. 
Our mechanism extends that of McAfee's in the SDA setting to an MDA market: price is formed collectively by all participating agents instead of being set by a third party, and the efficiency converges in a strong sense as the market grows large.

\section{THE MECHANISM}

In an MDA market with $m$ buyers and $n$ sellers, each buyer $i$ wants to purchase $X_{i}$ unit items and each seller $j$ has $Y_{j}$ unit items to sell. We assume both $X_{i}$ and $Y_{j}$ are public information, i.e., known to every agent. The reservation prices, which are private, for buyer $i$ and seller $j$ are $b_{i}$ and $s_{j}$, respectively. We assume the reservation price for each agent is static. Let $q_{i j}$ denote the quantity buyer $i$ buys from seller $j$, and $p_{i j}$ denote the trading price. Buyer $i$ 's utility for this transaction is then defined as $u b_{i}=\sum_{j=1}^{n}\left(b_{i}-p_{i j}\right) q_{i j}$, and seller $j$ 's utility is $u s_{j}=\sum_{i=1}^{m}\left(p_{i j}-s_{j}\right) q_{i j}$.

To induce the agents to report their true reservation prices, we apply a Vickrey-like auction on each side of the market. On the buyers' side, each buyer $i$ reports a price $r b_{i}$ (which may or may not equal $b_{i}$ ); and on the sellers' side, each seller $j$ reports a price $r s_{j}$ (which may or may not equal $s_{j}$ ). Without loss of generality, we assume

$$
r b_{1}>r b_{2} \ldots>r b_{m}
$$

and

$$
r s_{1}<r s_{2} \ldots<r s_{n} .
$$

We assume strict order relations here because if two buyers report the same reservation price, we can add their volumes together to form an equivalent bid. The same thing may be done with the sellers.

Our mechanism works as follows. It arranges the demand volumes according to the ascendent price order as shown in (1) and the supply volumes according to the decedent price order as shown in (2). At the critical point $q^{*}$ where the aggregate demand and supply meet, there are a buyer $K$ and a seller $L$. Either their reported prices satisfy

$$
r b_{K} \geq r s_{L} \geq r b_{K+1},
$$

and the aggregate demand and supply satisfy

$$
\sum_{1}^{L-1} Y_{j} \leq \sum_{1}^{K} X_{i} \leq \sum_{1}^{L} Y_{j}
$$

(Case I), or their reported prices satisfy

$$
r s_{L+1} \geq r b_{K} \geq r s_{L}
$$

and the aggregate demand and supply satisfy

$$
\sum_{1}^{K-1} X_{i} \leq \sum_{1}^{L} Y_{j} \leq \sum_{1}^{K} X_{i}
$$

(Case II).

We state our mechanism for Case I; Case II is similar. To clear the market, we first check whether inequality

$$
\sum_{1}^{K-1} X_{i} \geq \sum_{1}^{L-1} Y_{j}
$$

or

$$
\sum_{1}^{K-1} X_{i} \leq \sum_{1}^{L-1} Y_{j}
$$

holds. If (7) holds, we follow

rule1: all the sellers with indices $j<L$ sell all their volume $Y_{j}$ at price $r s_{L}$; all the buyers with indices $i<K$ trade at price $r b_{K}$ and each of them buys a volume equal to $X_{i}-\left(\sum_{1}^{K-1} X_{i}-\sum_{1}^{L-1} Y_{j}\right) /(k-1)^{1}$.

If (8) holds, we follow

rule2: all the buyers with indices $i<K$ buy all their volume $X_{i}$ at price $r b_{K}$; all the sellers with indices $j<L$ trade at price $r s_{L}$ and each of them sells a volume equal to $Y_{j}-\left(\sum_{1}^{L-1} Y_{j}-\sum_{1}^{K-1} X_{i}\right) /(L-1)$.

If (7) holds, there is a over-demand, and this over-demand is averaged over all the first $K-1$ buyers; If (8) holds, there is a over-supply, and this over-supply is split over all the first $L-1$ sellers.

The total trade volume is $\min \left(\sum_{1}^{K-1} X_{i}, \quad \sum_{1}^{L-1} Y_{j}\right)$.

The trading surplus $\left(r b_{K}-r s_{L}\right) \min \left(\sum_{1}^{K-1} X_{i}, \sum_{1}^{L-1} Y_{j}\right)$ is taken by the market maker. The potential trading value between buyer $K$ and seller $L$ is sacrificed, not collected by any of the three parties, the sellers, the buyers or the market maker, involved in the market. Since either buyers or sellers are forced to sacrifice part of their volumes, there is yet another potential trading value lost. If (7) holds, this loss is bounded by $\left(r b_{1}-r b_{K}\right) Y_{L}$, and if (8) holds, this loss is bounded by $\left(r s_{L}-r s_{1}\right) X_{K}$.

To summarize, our mechanism is as follows:

Mechanism: The market maker first sorts the reported reservation prices from the buyers and sellers according to inequalities (1) and (2). Every buyer with index less than $K$ and every seller with index less than $L$ will trade. Then depending on whether the market situation is Case I or Case II, and whether inequality (7) or (8) holds, the market maker decides whether to apply rule1 or rule2.

Theorem 1: Under the assumption that the buyers and sellers' volumes are public information, the above mechanism is strategy-proof with respect to reservation price, weakly budget-balanced, and individually rational.

Proof: See [2] for details.

\section{REFERENCES}

[1] S. Barbera and M. O. Jackson. Strategy-proof exchange. Econometrica, 63(1):51-87, 1995.

[2] P. Hunag, A. Scheller-Wolf, and K. Sycara. A strategy-proff multiunit double auction mechanism (extended version). Working Paper, Carnegie Mellon University, 2001.

[3] R. P. McAfee. A dominant strategy double auction. Journal of Economic Theory, 56:434-450, 1992.

[4] M. A. Satterthwaite and S. R. Williams. The rate of convergence to efficiency in the buyer's bid double auction as the market becomes large. The Review of Economic Studies, 56(4):477-498, 1989.

\footnotetext{
${ }^{1}$ If $\left(\sum_{1}^{K-1} X_{i}-\sum_{1}^{L-1} Y_{j}\right) /(k-1)>X_{i}$ for some buyer $i$, this buyer buys nothing and the "burden left", $\left(\sum_{1}^{K-1} X_{i}-\right.$ $\left.\sum_{1}^{L-1} Y_{j}\right) /(k-1)-X_{i}$, is averaged over the $K-2$ buyers left. Continue this procedure until each buyer left trades a positive volume. A similar procedure may also apply to rule2.
} 